\title{
Thermoelastic Problem of a Long Annular Multilayered Cylinder
}

\author{
Yi Hsien $\mathrm{Wu}^{{ }^{*}}$, Kuo-Chang Jane ${ }^{2}$ \\ ${ }^{1}$ Department of Information Management, Oriental Institute of Technology, Taipei, Chinese Taipei \\ ${ }^{2}$ Department of Applied Mathematics, National Chung Hsing University, Taichung, Chinese Taipei \\ Email: *yhwu@mail.oit.edu.tw
}

Received May 2, 2013; revised June 2, 2013; accepted June 9, 2013

Copyright @ 2013 Yi Hsien Wu, Kuo-Chang Jane. This is an open access article distributed under the Creative Commons Attribution License, which permits unrestricted use, distribution, and reproduction in any medium, provided the original work is properly cited.

\begin{abstract}
Thermoelastic transient response of multilayered annular cylinders of infinite lengths subjected to known inner pressure and outer surfaces cooling are considered. A method based on the Laplace transformation and finite difference method has been developed to analyze the thermoelasticity problem. Using the Laplace transform with respect to time, the general solutions of the governing equations are obtained in transform domain. The solution is obtained by using the matrix similarity transformation and inverse Laplace transform. Solutions for the temperature and thermal stress distributions in a transient state were obtained. It was found that the temperature distribution, the displacement and the thermal stresses change slightly as time increases.
\end{abstract}

Keywords: Thermoelastic; Multilayered Annular Cylinders; Laplace Transformation; Finite Difference Method

\section{Introduction}

A thermal problem arises when the composed materials are generated by a sudden change in temperature. Shell structures are widely used in contemporary industries, so we must take care of the thermal problem. The shell structures may be affected due to the pressure change or the various temperature distributions. It is necessary to solve for temperature or pressure at first.

The dynamic thermoelastic response of circular shell rapidly change of thermal environments is important for the design of many engineering structures. Due to the complexity of the governing equations and the mathematical difficulties associated with the solution, several simplifications have been used. For example, Sherief and Anwar [1] discussed the problem of an annular infinitely long elastic circular. They have neglected both the inertia terms and the relaxation effects of the problem. Sherief and Anwar [2] considered the thermoelasticity problem of an infinitely long annular cylinder composed of two different materials with axial symmetry. The solution was obtained in the Laplace transform domain by using the potential function approach.

The present work deals with the one-dimensional quasistatic coupled thermoelastic problems of an infinitely long annular multilayered cylinder composed of multi-

\footnotetext{
"Corresponding author.
}

layered different materials. The medium has a pressure at the inner layer, the temperature to be heated at the outer layer, without body forces and internal heat generation. Derivatives are approximated by central differences resulting in an algebraic representation of the partial differential equation. By taking the Laplace transform with respect to time, the general solutions in the transform domain are first obtained. The final solutions in the real domain can be obtained by inverting the Laplace transform.

\section{Formulation}

This work deals with the one-dimensional, quasi-static coupled, thermoelastic problems of an infinitely long annular cylinder composed of multilayered laminated materials with axial symmetry under the following assumptions: 1) Materials of each layer are assumed to be non-homogeneous; 2) Deformation and strain satisfy the Hooke's law and small strain theory; 3) The composite cylinder is constructed of multilayered laminates bonded together perfectly; 4) The medium is initially undisturbed, and without body forces and internal heat sources; 5) The medium is applied by a force, which is the function of time; 6) The temperature at inner layer and outer layer are the functions of time.

We now consider an infinitely long annular cylinder 
made of multiple layers of different materials. The inner and outer radii of the cylinder are denoted by $r_{i}$ and $r_{o}$, respectively. The multilayered composite is assumed to be heated suddenly at the inner and outer surface under temperatures $f_{1}$ and $f_{2}$ respectively.

The transient heat conduction equation for the ith layer in dimensional form can be written as (see Equation (1) below)

where

$$
\bar{\Theta}=\Theta-\Theta_{0} \quad \beta_{r}=\frac{E_{r}}{1-v_{r \theta} v_{\theta r}}\left(\alpha_{r}+v_{\theta r} \alpha_{\theta}\right)
$$

and

$$
\beta_{r}=\frac{E_{r}}{1-v_{r \theta} v_{\theta r}}\left(\alpha_{\theta}+v_{\theta r} \alpha_{r}\right)
$$

in which $U$ is the radial component of displacement, $r^{*}$ is radius, $C_{v}$ and $\rho$ are specific heat and density of material, $v_{\theta r}$ and $v_{r \theta}$ are the Poisson's ratio $k_{r}$, $k_{\theta}$ are radial, circumferential thermal conductivity, $\alpha_{r}$, $\alpha_{\theta}$ are radial and circumferential thermal expansion coefficient, $E_{r}, E_{\theta}$ are radial and circumferential Young's modulus, $\Theta, \Theta_{0}$ are the temperature, reference temperature, and $\tau$ is time, respectively.

If the body forces are absent, the equation of equilibrium for a cylinder along the radial direction can be written as

$$
\begin{aligned}
& \frac{\partial^{2} U}{\partial r^{*^{2}}}+\left[\frac{E_{\theta}}{E_{r}} v_{\theta r}+\left(1-v_{r \theta}\right)\right] \frac{1}{r^{*}} \frac{\partial U}{\partial r^{*}}-\frac{E_{\theta}}{E_{r}} \frac{1}{r^{*^{2}}} U \\
& =\left(\alpha_{r}+v_{\theta r} \alpha_{\theta}\right) \frac{\partial \bar{\Theta}}{\partial r^{*}}-\left[\frac{E_{\theta}}{E_{r}}\left(\alpha_{\theta}+v_{r \theta} \alpha_{r}\right)-\left(\alpha_{r}+v_{\theta r} \alpha_{\theta}\right)\right] \frac{\bar{\Theta}}{r^{*}}
\end{aligned}
$$

The stress-displacement relations are

$$
\begin{gathered}
\sigma_{r i}^{*}=\left(\frac{E_{r}}{\lambda}\right)_{i} \frac{\partial U}{\partial r^{*}}+\left(\frac{E_{\theta} v_{r \theta}}{\lambda}\right)_{i} \frac{U}{r^{*}}-\beta_{r i}\left(\Theta-\Theta_{0}\right) \\
\sigma_{\theta i}^{*}=\left(\frac{E_{r} v_{r \theta}}{\lambda}\right)_{i} \frac{\partial U}{\partial r^{*}}+\left(\frac{E_{\theta}}{\lambda}\right)_{i} \frac{U}{r^{*}}-\beta_{\theta i}\left(\Theta-\Theta_{0}\right)
\end{gathered}
$$

where $\lambda, \sigma_{r}^{*}, \sigma_{\theta}^{*}$ are Lame's constant, radial and circumferential stresses respectively.

Let the boundary conditions of multilayered cylinder be at

$$
t=0 \quad U=\bar{\Theta}=0
$$

at

$$
r^{*}=R_{1} \quad \sigma_{r}^{*}(r, t)=-P_{0} \mathrm{e}^{-c_{1} t} \quad \Theta_{1}-\Theta_{0}=f_{1}
$$

at

$$
r^{*}=R_{o} \quad \sigma_{r}^{*}(r, t)=0 \quad \Theta_{\text {out }}-\Theta_{0}=f_{2}=\Theta_{r}\left(1-\mathrm{e}^{-c_{2} t}\right)
$$

where $f_{1}, f_{2}, P_{0}, \Theta_{r}$ are inner and outer surrounding temperatures, initial inner pressure, the initial temperature at the outer layer respectively.

The non-dimensional variables are defined as follows:

$$
\begin{aligned}
& T=\left(\Theta-\Theta_{0}\right) / \Theta_{0}=\bar{\Theta} / \Theta_{0} \\
& a_{i}=\left(\frac{k_{r} \cos ^{2} \theta+k_{\theta} \sin ^{2} \theta}{\rho C_{v}}\right)_{i} /\left(\frac{k_{r} \cos ^{2} \theta+k_{\theta} \sin ^{2} \theta}{\rho C_{v}}\right)_{1} \\
& b_{i}=\left(\frac{k_{r} \sin ^{2} \theta+k_{\theta} \cos ^{2} \theta}{\rho C_{v}}\right)_{i} /\left(\frac{k_{r} \cos ^{2} \theta+k_{\theta} \sin ^{2} \theta}{\rho C_{v}}\right)_{1} \\
& w_{i}=\left(\frac{\beta_{r} \sin ^{2} \theta+\beta_{\theta} \cos ^{2} \theta}{\rho C_{v}}\right)_{i} /\left(\frac{\beta_{r} \cos ^{2} \theta+\beta_{\theta} \sin ^{2} \theta}{\rho C_{v}}\right)_{i} \\
& t=\tau\left(\frac{k_{r} \cos ^{2} \theta+k_{\theta} \sin ^{2} \theta}{\rho C_{v}}\right)_{1} / R_{1}^{2} \quad r=r^{*} / R_{1} \\
& u=U\left(\frac{\beta_{r} \cos ^{2} \theta+\beta_{\theta} \sin ^{2} \theta}{\rho C_{v}}\right)_{i} / R_{1} \\
& e_{i}=\left[v_{\theta r} E_{\theta} / E_{r}+\left(1-v_{r \theta}\right)\right]_{i} \quad f_{i}=\left(E_{\theta} / E_{r}\right)_{i} \\
& g_{i}=\left(\frac{\beta_{r} \cos ^{2} \theta+\beta_{\theta} \sin ^{2} \theta}{\rho C_{v}}\right)_{i}\left(\alpha_{r}+v_{\theta r} \alpha_{\theta}\right)_{i} \Theta_{0} \\
& h_{i}=\left(\frac{\beta_{r} \cos ^{2} \theta+\beta_{\theta} \sin ^{2} \theta}{\rho C_{v}}\right)_{i}\left[\frac{E_{\theta}}{E_{r}}\left(\alpha_{\theta}+v_{r \theta} \alpha_{r}\right)-\left(\alpha_{r}+v_{\theta r} \alpha_{\theta}\right)\right]_{i} \Theta_{0} \\
& { }_{1} Q_{i}=\left(\frac{E_{r}}{\lambda}\right)_{i} /\left[\beta_{r 1}\left(\frac{\beta_{r} \cos ^{2} \theta+\beta_{\theta} \sin ^{2} \theta}{\rho C_{v}}\right)_{i} \Theta_{0}\right] \\
& { }_{2} Q_{i}=\left(\frac{E_{\theta} v_{\theta r}}{\lambda}\right)_{i} /\left[\beta_{r 1}\left(\frac{\beta_{r} \cos ^{2} \theta+\beta_{\theta} \sin ^{2} \theta}{\rho C_{v}}\right)_{i} \Theta_{0}\right] \\
& { }_{3} Q_{i}=\beta_{r i} / \beta_{r 1} \\
& { }_{1} R_{i}=\left(\frac{E_{r} v_{r \theta}}{\lambda}\right)_{i} /\left[\beta_{\theta 1}\left(\frac{\beta_{r} \cos ^{2} \theta+\beta_{\theta} \sin ^{2} \theta}{\rho C_{v}}\right)_{i} \Theta_{0}\right] \\
& { }_{2} R_{i}=\left(\frac{E_{r}}{\lambda}\right)_{i} /\left[\beta_{\theta 1}\left(\frac{\beta_{r} \cos ^{2} \theta+\beta_{\theta} \sin ^{2} \theta}{\rho C_{v}}\right)_{i} \Theta_{0}\right]
\end{aligned}
$$

$$
\begin{aligned}
{\left[\left(k_{r} \cos ^{2} \theta+k_{\theta} \sin ^{2} \theta\right) \frac{\partial^{2}}{\partial r^{*^{2}}}+\left(k_{r} \sin ^{2} \theta+k_{\theta} \cos ^{2} \theta\right) \times \frac{1}{r^{*}} \frac{\partial}{\partial r^{*}}\right] \bar{\Theta}=} & \rho C_{v} \frac{\partial \bar{\Theta}}{\partial \tau}+\Theta_{0}\left(\beta_{r} \cos ^{2} \theta+\beta_{\theta} \sin ^{2} \theta\right) \frac{\partial}{\partial r^{*}}\left(\frac{\partial U}{\partial \tau}\right) \\
& +\Theta_{0}\left(\beta_{r} \sin ^{2} \theta+\beta_{\theta} \cos ^{2} \theta\right) \frac{1}{r^{*}}\left(\frac{\partial U}{\partial \tau}\right)
\end{aligned}
$$




$$
{ }_{3} R_{i}=\beta_{\theta i} / \beta_{\theta 1} \quad \sigma_{r i}=\sigma_{r i}^{*} /\left(\beta_{r 1} \Theta_{0}\right) \quad \sigma_{\theta i}=\sigma_{\theta i}^{*} /\left(\beta_{\theta 1} \Theta_{0}\right)
$$

where $T, t, r, u, \sigma_{r i}, \sigma_{\theta i}$ are non-dimensional temperature, time, radius, displacement, radial stress and circumferential stress for the ith layer respectively.

Substituting the nondimensional quantities into the governing Equations (1)-(4), the transient heat conduction equation and stress-displacement relations have the following nondimensional form:

$$
\left\{\left[a_{i} \frac{\partial^{2}}{\partial r^{2}}+\frac{b_{i}}{r} \frac{\partial}{\partial r}\right]-\frac{\partial}{\partial t}\right\} T=\frac{\partial}{\partial r}\left(\frac{\partial u}{\partial t}\right)+\frac{w_{i}}{r}\left(\frac{\partial u}{\partial t}\right)
$$

$$
\begin{gathered}
\frac{\partial^{2} u}{\partial r^{2}}+\frac{e_{i}}{r} \frac{\partial u}{\partial r}-f_{i} \frac{u}{r^{2}}=g_{i} \frac{\partial T}{\partial r}-h_{i} \frac{T}{r} \\
\sigma_{r i}={ }_{1} Q_{i} \frac{\partial u}{\partial r}+{ }_{2} Q_{i} \frac{u}{r}-{ }_{3} Q_{i} T \\
\sigma_{\theta i}={ }_{1} R_{i} \frac{\partial u}{\partial r}+{ }_{2} R_{i} \frac{u}{r}-{ }_{3} R_{i} T
\end{gathered}
$$

\section{Computational Procedures}

Applying central difference in Equations (5)-(8), we arrive at the following discretized equations:

$$
\begin{gathered}
a_{i} \frac{T_{j+1}-2 T_{j}+T_{j-1}}{\left(\Delta r_{j}\right)^{2}}+b_{i} \frac{1}{r_{j}} \frac{T_{j+1}-T_{j-1}}{2 \Delta r_{j}}-\frac{\partial T_{j}}{\partial t}=\frac{w_{i}}{r_{j}} \frac{\partial u_{j}}{\partial t}+\frac{\left(\frac{\partial u}{\partial t}\right)_{j+1}-\left(\frac{\partial u}{\partial t}\right)_{j-1}}{2 \Delta r_{j}} \\
\frac{u_{j+1}-2 u_{j}+u_{j-1}}{\left(\Delta r_{j}\right)^{2}}+e_{i} \frac{1}{r_{j}} \frac{u_{j+1}-u_{j-1}}{2 \Delta r_{j}}-f_{i} \frac{1}{r_{j}^{2}} u_{j}=g_{i} \frac{T_{j+1}-T_{j-1}}{2 \Delta r_{j}}-h_{i} \frac{T_{j}}{r_{j}}
\end{gathered}
$$$$
\sigma_{r i}={ }_{1} Q_{i} \frac{u_{j+1}-u_{j-1}}{2 \Delta r_{j}}+{ }_{2} Q_{i} \frac{u_{j}}{r_{j}}-{ }_{3} Q_{i} T_{j}
$$$$
\sigma_{\theta i}={ }_{1} R_{i} \frac{u_{j+1}-u_{j-1}}{2 \Delta r_{j}}+{ }_{2} R_{i} \frac{u_{j}}{r_{j}}-{ }_{3} R_{i} T_{j}
$$

where

$$
\begin{aligned}
& \begin{aligned}
& \Delta r_{j}=1 /(N-1) \text { obtain the following equations: } \\
& a_{i} \frac{\bar{T}_{j+1}-2 \bar{T}_{j}+\bar{T}_{j-1}}{\left(\Delta r_{j}\right)^{2}}+b_{i} \frac{1}{r_{j}} \frac{\bar{T}_{j+1}-\bar{T}_{j-1}}{2 \Delta r_{j}}-\left(T_{j, i n}+s \bar{T}_{j}\right)= \frac{w_{i}}{r_{j}}\left(u_{j, i n}+s \bar{u}_{j}\right) \\
&+\frac{1}{2 \Delta r_{j}}\left[\left(u_{j+1, i n}+s \bar{u}_{j+1}\right)-\left(u_{j-1, i n}+s \bar{u}_{j-1}\right)\right] \\
& \frac{\bar{u}_{j+1}-2 \bar{u}_{j}+\bar{u}_{j-1}}{\left(\Delta r_{j}\right)^{2}}+e_{i} \frac{1}{r_{j}} \frac{\bar{u}_{j+1}-\bar{u}_{j-1}}{2 \Delta r_{j}}-f_{i} \frac{1}{r_{j}^{2}} \bar{u}_{j}=g_{i} \frac{\bar{T}_{j+1}-\bar{T}_{j-1}}{2 \Delta r_{j}}-h_{i} \frac{\bar{T}_{j}}{r_{j}}
\end{aligned}
\end{aligned}
$$

Let the surface of the cylindrical inner surface be stress free and subject to a time-dependent temperature. After taking Laplace transformation, the boundary conditions in transformed domain become

$$
\bar{\sigma}_{r}(r, s)=\frac{-P_{0}}{\beta_{r 1} \Theta_{0}} \frac{1}{s+c_{1}} \quad \bar{T}_{1}=\bar{f}_{1}(s) / \Theta_{0}
$$

at $r=r_{1}$;

$$
\bar{\sigma}_{r}(r, s)=0 \quad \bar{T}_{1}=\Theta_{r}\left(\frac{1}{s}-\frac{1}{s+c_{2}}\right)
$$

at $r=r_{\text {out }}$. and $j=1,2, \cdots, N$.

The Laplace transform of a function $\Phi(t)$ are defined by

$$
\bar{\Phi}(s)=L[\Phi(t)]=\int_{0}^{\infty} \mathrm{e}^{-s t} \Phi(t) \mathrm{d} t
$$

Take the Laplace transform for Equations (9)-(12), we

and the interface conditions are as follows:

$$
\begin{array}{ll}
\bar{u}_{i}(r, s)=\bar{u}_{i+1}(r, s) & r=r_{i+1} \\
\bar{\sigma}_{r i}(r, s)=\bar{\sigma}_{r i+1}(r, s) & r=r_{i+1} \\
\bar{q}_{i}(r, s)=\bar{q}_{i+1}(r, s) & r=r_{i+1} \\
\bar{T}_{i}(r, s)=\bar{T}_{i+1}(r, s) & r=r_{i+1}
\end{array}
$$

where

$$
i=1,2, \cdots, m-1 \text {. }
$$

Substituting the boundary conditions and the interface conditions into Equations (13), (14), we obtain the fol- 
lowing equation in matrix form (see Equations (15) below)

where

$$
\begin{aligned}
& B_{1}=-\left[1+\frac{{ }_{3} Q_{1}}{{ }_{1} Q_{1}}\right]^{-1}\left[\frac{2 a_{1}}{\left(\Delta r_{1}\right)^{2}}\right] \\
& B_{j}=-\frac{2 a_{i}}{\left(\Delta r_{i}\right)^{2}} \\
& E_{1}=\left[1+\frac{{ }_{3} Q_{1}}{{ }_{1} Q_{1}}\right]^{-1}\left[\frac{1}{r_{1}} \frac{{ }_{2} Q_{1} Q_{1}}{{ }_{1}}-\frac{w_{1}}{r_{1}}\right] \\
& C_{j}=\frac{a_{i}}{\left(\Delta r_{i}\right)^{2}}+\frac{b_{i}}{r_{j}} \frac{1}{2 \Delta r_{i}} \\
& F_{1}=D_{N}=\bar{X}_{N}=\bar{Y}_{1}=\bar{Z}_{1}=\bar{G}_{N}=0 \quad D_{j}=\frac{1}{2 \Delta r_{i}} \\
& A_{N}=\left[1+\frac{{ }_{3} Q_{m}}{{ }_{1} Q_{m}}\right]^{-1}\left[\frac{a_{m}}{\left(\Delta r_{m}\right)^{2}}-\frac{b_{m}}{r_{k}} \frac{1}{2 \Delta r_{m}}\right] \\
& E_{j}=-\frac{w_{i}}{r_{j}} \\
& B_{N}=-\left[1+\frac{{ }_{3} Q_{m}}{{ }_{1} Q_{m}}\right]^{-1}\left[\frac{2 a_{m}}{\left(\Delta r_{m}\right)^{2}}\right] \\
& F_{j}=-\frac{1}{2 \Delta r_{i}} \\
& E_{N}=\left[1+\frac{{ }_{3} Q_{m}}{{ }_{1} Q_{m}}\right]^{-1}\left[-\frac{w_{m}}{r_{k}}+\frac{{ }_{2} Q_{m}}{{ }_{1} Q_{m}} \frac{1}{r_{k}}\right] \quad \bar{X}_{j}=0
\end{aligned}
$$

$$
\bar{X}_{1}=\left[1+\frac{{ }_{3} Q_{1}}{{ }_{1} Q_{1}}\right]^{-1} \frac{P_{0}}{{ }_{1} Q_{1}} \quad \bar{Y}_{j}=0
$$

$$
\begin{gathered}
\bar{Y}_{N}=\left[1+\frac{{ }_{3} Q_{2}}{{ }_{1} Q_{2}}\right]^{-1}\left[\left(\frac{a_{m}}{\left(\Delta r_{m}\right)^{2}}+\frac{b_{m}}{r_{k}} \frac{1}{2 \Delta r_{m}}\right) \Theta_{r}\right] \bar{Z}_{j}=0 \\
\bar{Z}_{N}=\left[1+\frac{{ }_{3} Q_{2}}{{ }_{1} Q_{2}}\right]^{-1}\left[\left(\frac{a_{m}}{\left(\Delta r_{m}\right)^{2}}+\frac{b_{m}}{r_{k}} \frac{1}{2 \Delta r_{m}}\right) \Theta_{r}\right] \bar{G}_{j}=0 \\
\bar{G}_{1}=\left[1+\frac{{ }_{3} Q_{1}}{{ }_{1} Q_{1}}\right]^{-1}\left\{\left[\frac{b_{1}}{r_{1}} \frac{1}{2 \Delta r_{1}}-\frac{a_{1}}{\left(\Delta r_{1}\right)^{2}}\right] T_{1}-\frac{P_{0}}{{ }_{1} Q_{1}}\right\}
\end{gathered}
$$

where $m$ denotes the last layer, $k$ the last point, and $i$ denotes ith layer for

$$
j=2,3, \cdots, N-1 \text {, }
$$

(see Equation (16) below)

where:

$$
\begin{gathered}
H_{j}=\frac{g_{i}}{2 \Delta r_{i}} \quad I_{j}=\frac{h_{i}}{r_{j}} \quad J_{j}=-\frac{g_{i}}{2 \Delta r_{i}} \\
K_{j}=\frac{1}{\left(\Delta r_{i}\right)^{2}}-\frac{e_{i}}{r_{j}} \frac{1}{2 \Delta r_{i}} \\
L_{j}=-\left[\frac{2}{\left(\Delta r_{i}\right)^{2}}+\frac{f_{i}}{r_{j}^{2}}\right] \\
M_{j}=\frac{1}{\left(\Delta r_{i}\right)^{2}}+\frac{e_{i}}{r_{j}} \frac{1}{2 \Delta r_{i}} \\
j=2,3, \cdots, N-1
\end{gathered}
$$

$$
\begin{aligned}
& \left\{\left[\begin{array}{ccccc}
B_{1} & C_{1} & & & \\
A_{2} & B_{2} & C_{2} & & \\
& \ddots & \ddots & \ddots & \\
& & A_{N-1} & B_{N-1} & C_{N-1} \\
& & & A_{N} & B_{N}
\end{array}\right]-s[I]\right\}\left[\begin{array}{c}
\bar{T}_{1} \\
\bar{T}_{2} \\
\vdots \\
\bar{T}_{N-1} \\
\bar{T}_{N}
\end{array}\right]-\frac{1}{s+C_{1}}\left[\begin{array}{c}
\bar{X}_{1} \\
\bar{X}_{2} \\
\vdots \\
\bar{X}_{N-1} \\
\bar{X}_{N}
\end{array}\right]-\frac{1}{s+C_{2}}\left[\begin{array}{c}
\bar{Y}_{1} \\
\bar{Y}_{2} \\
\vdots \\
\bar{Y}_{N-1} \\
\bar{Y}_{N}
\end{array}\right]+\frac{1}{S}\left[\begin{array}{c}
\bar{Z}_{1} \\
\bar{Z}_{2} \\
\vdots \\
\bar{Z}_{N-1} \\
\bar{Z}_{N}
\end{array}\right] \\
& +S\left[\begin{array}{ccccc}
E_{1} & F_{1} & & & \\
D_{2} & E_{2} & F_{2} & & \\
& \ddots & \ddots & \ddots & \\
& & D_{N-1} & E_{N-1} & F_{N-1} \\
& & & D_{N} & E_{N}
\end{array}\right]\left[\begin{array}{c}
\bar{u}_{1} \\
\bar{u}_{2} \\
\vdots \\
\bar{u}_{N-1} \\
\bar{u}_{N}
\end{array}\right]=\left[\begin{array}{c}
\bar{G}_{1} \\
\bar{G}_{2} \\
\vdots \\
\bar{G}_{N-1} \\
\bar{G}_{N}
\end{array}\right] \\
& {\left[\begin{array}{ccccc}
I_{1} & J_{1} & & & \\
H_{2} & I_{2} & J_{2} & & \\
& \ddots & \ddots & \ddots & \\
& & H_{N-1} & I_{N-1} & J_{N-1} \\
& & & H_{N} & I_{N}
\end{array}\right]\left[\begin{array}{c}
\bar{T}_{1} \\
\bar{T}_{2} \\
\vdots \\
\bar{T}_{N-1} \\
\bar{T}_{N}
\end{array}\right]+\left[\begin{array}{ccccc}
L_{1} & M_{1} & & \\
K_{2} & L_{2} & M_{2} & & \\
& \ddots & \ddots & \ddots & \\
& & K_{N-1} & L_{N-1} & M_{N-1} \\
& & & K_{N} & L_{N}
\end{array}\right]\left[\begin{array}{c}
\bar{u}_{1} \\
\bar{u}_{2} \\
\vdots \\
\bar{u}_{N-1} \\
\bar{u}_{N}
\end{array}\right]=\left[\begin{array}{c}
0 \\
0 \\
\vdots \\
0 \\
0
\end{array}\right]}
\end{aligned}
$$


Equations (15) and (16) can be rewritten in the following matrix forms

$$
\begin{gathered}
\{[M]-s[I]\}\left\{\bar{T}_{j}\right\}+s[N]\left\{\bar{u}_{j}\right\}-\frac{1}{s+c_{1}}\left\{\bar{X}_{j}\right\} \\
-\frac{1}{s+c_{2}}\left\{\bar{Y}_{j}\right\}+\frac{1}{s}\left\{\bar{Z}_{j}\right\}=\left\{\bar{G}_{j}\right\} \\
{[R]\left\{\bar{T}_{j}\right\}+[Q]\left\{\bar{u}_{j}\right\}=0}
\end{gathered}
$$

where the matrix $[M],[N],[R]$ and $[Q]$ are the corresponding matrix in Equations (15) and (16). Substituting Equation (17) into (18), we have

$$
\begin{aligned}
& \{[A]-s[I]\}\left\{\bar{T}_{j}\right\}-\frac{1}{s+c_{1}}\left\{\bar{B}_{j}\right\} \\
& -\frac{1}{s+c_{2}}\left\{\bar{C}_{j}\right\}+\frac{1}{s}\left\{\bar{D}_{j}\right\}=\left\{\bar{F}_{j}\right\}
\end{aligned}
$$

where

$$
\begin{aligned}
{[A] } & =\left\{[N]^{-1}+[Q]^{-1}[R]\right\}^{-1}[N]^{-1}[M] \\
\left\{\bar{B}_{j}\right\} & =\left\{[N]^{-1}+[Q]^{-1}[R]\right\}^{-1}[N]^{-1}\left\{\bar{X}_{j}\right\} \\
\left\{\bar{C}_{j}\right\} & =\left\{[N]^{-1}+[Q]^{-1}[R]\right\}^{-1}[N]^{-1}\left\{\bar{Y}_{j}\right\} \\
\left\{\bar{D}_{j}\right\} & =\left\{[N]^{-1}+[Q]^{-1}[R]\right\}^{-1}[N]^{-1}\left\{\bar{Z}_{j}\right\} \\
\left\{\bar{F}_{j}\right\} & =\left\{[N]^{-1}+[Q]^{-1}[R]\right\}^{-1}[N]^{-1}\left\{\bar{G}_{j}\right\}
\end{aligned}
$$

Since the $(N \times N)$ matrix $[A]$ is a nonsingular real matrix, the matrix $[A]$ possesses a set of $N$ linearly independent eigenvectors, hence the matrix $[A]$ is diagonalizable. There exist a nonsingular transition matrix $[P]$ such that $[P]^{-1}[A][P]=\operatorname{diag}[A]$, that is, the matrices $[A]$ and $\operatorname{diag}[A]$ are similar, where the matrix $\operatorname{diag}[A]$ is a diagonal matrix with elements $\lambda_{j}(j=1,2, \cdots, N)$, where $\lambda_{j}$ is the eigenvalue of matrix $[A]$.

The equation can be obtained as

$$
\begin{aligned}
& \left\{[P]^{-1}[A][P]-s[I]\right\}[P]^{-1}\left\{\bar{T}_{j}\right\}-\frac{1}{s+c_{1}}[P]^{-1}\left\{\bar{B}_{j}\right\} \\
& -\frac{1}{s+c_{2}}[P]^{-1}\left\{\bar{C}_{j}\right\}+\frac{1}{s}[P]^{-1}\left\{\bar{D}_{j}\right\}=[P]^{-1}\left\{\bar{F}_{j}\right\}
\end{aligned}
$$

Equation (20) can be rewritten as

$$
\begin{aligned}
& \{\operatorname{diag}[A]-s[I]\}\left\{\bar{T}_{j}^{*}\right\}-\frac{1}{s+c_{1}}\left\{\bar{B}_{j}^{*}\right\} \\
& -\frac{1}{s+c_{2}}\left\{\bar{C}_{j}^{*}\right\}+\frac{1}{s}\left\{\bar{D}_{j}^{*}\right\}=\left\{\bar{F}_{j}^{*}\right\}
\end{aligned}
$$

where

$$
\begin{gathered}
\left\{\bar{T}_{j}^{*}\right\}=[P]^{-1}\left\{\bar{T}_{j}\right\}, \quad\left\{\bar{B}_{j}^{*}\right\}=[P]^{-1}\left\{\bar{B}_{j}\right\} \\
\left\{\bar{C}_{j}^{*}\right\}=[P]^{-1}\left\{\bar{C}_{j}\right\}, \quad\left\{\bar{D}_{j}^{*}\right\}=[P]^{-1}\left\{\bar{D}_{j}\right\}
\end{gathered}
$$

and

$$
\left\{\bar{F}_{j}^{*}\right\}=[P]^{-1}\left\{\bar{F}_{j}\right\}
$$

From Equation (21), the following solutions can be obtained immediately.

$$
\bar{T}_{j}^{*}=\frac{\bar{F}_{j}^{*}}{\lambda_{i}-s}+\frac{\bar{B}_{j}^{*}}{\left(s+c_{1}\right)\left(\lambda_{i}-s\right)}+\frac{\bar{C}_{j}^{*}}{\left(s+c_{2}\right)\left(\lambda_{i}-s\right)}-\frac{\bar{D}_{j}^{*}}{s\left(\lambda_{i}-s\right)}
$$

By applying the inverse Laplace transforms to Equation (22), we get the solution $T_{j}^{*}$. The eigenvalue, eigenvector and inverse Laplace transform of matrix $[A]$ can be solved by applying the IMSL MATH/LIBRARY subroutines.

After we have obtained $T_{j}^{*}$, then we can use Equations (23) and (24) to obtain the solutions $T_{j}$ and $u_{j}$

$$
\begin{gathered}
\left\{T_{j}\right\}=[P]\left\{T_{j}^{*}\right\} \\
\left\{u_{j}\right\}=-[Q]^{-1}[R]\left\{T_{j}\right\}
\end{gathered}
$$

Substituting $T_{j}$ and $u_{j}$ into Equations (11) and (12), we obtain the radial and circumferential stresses.

\section{Numerical Results and Discussions}

In this section, we present some numerical results of the temperature distribution in a long multilayered composite hollow cylinder, and displacement and thermal stresses under temperature changes.

The inner and outer radii of the cylinder are assumed to be 1.0 and 4.5 respectively. For an infinitely long annular multilayered cylinder, the geometry and material quantities of the cylinder (in the case of three layers, layer 1: $E=58 \mathrm{E} 6, k=22, v=0.2, \alpha=2.8 \mathrm{E}-6, \rho=0.095$, $C_{v}=0.31$ and layer $2: E=30 \mathrm{E} 6, k=21, v=0.35, \alpha=$ 2.3E $-6, \rho=0.053, C_{v}=0.25$ and layer $3: E=22 \mathrm{E} 6, k=$ $17, v=0.2, \alpha=2.8 \mathrm{E}-6, \rho=0.09, C_{v}=0.17$; in the case of five layers, layer $1: E=58 \mathrm{E} 6, k=22, v=0.2, \alpha=$ 2.8E - 6, $\rho=0.095, C_{v}=0.31$ and layer $2: E=30 \mathrm{E} 6, k=$ $21, v=0.35, \alpha=2.3 \mathrm{E}-6, \rho=0.053, C_{v}=0.25$ and layer $3: E=22 \mathrm{E} 6, k=17, v=0.2, \alpha=2.8 \mathrm{E}-6, \rho=0.09, C_{v}=$ 0.17 and layer 4: $E=30 \mathrm{E} 6, k=21, v=0.35, \alpha=2.3 \mathrm{E}-6$, $\rho=0.053, C_{v}=0.25$ and layer $5: E=22 \mathrm{E} 6, k=17, v=$ $\left.0.2, \alpha=2.8 \mathrm{E}-6, \rho=0.09, C_{v}=0.17\right)$. Each layer is assumed to have a different thickness (in the case of three layers, $r_{1}=1.5, r_{2}=0.5$ and $r_{3}=1.5$; in the case of five layers, $r_{1}=1.0, r_{2}=0.5, r_{3}=1.0, r_{4}=0.5$ and $r_{5}=0.5$ ). The pressure of the inner surface is assumed to be $P_{0}=$ 1.5E6. The constant coefficient $c_{1}=c_{2}=1.0$. The temperature at inner surface is assumed to be 300 , at outer 
surface which is a function of time is assumed to be 0 to 100. Figures 1-4 show some numerical results of three and five layered cylinders at time step $t=0.5,1,2,5$ and 10.

Figures 1 and $\mathbf{2}$ show the temperature distributions along radial direction for 3 and 5 layers case. Because of the difference in thermal conductivity and the effect of the outer layer is to be heated. As time is small, say $t=0.5$, the outer layer temperature which is to be heated is not so more, so the distribution decreasing at first and then increasing. Figures $\mathbf{3}$ and $\mathbf{4}$ show the displacement along the radial direction. The maximum displacement occurred at the interface of first and second layers. Figures $\mathbf{5}$ and $\mathbf{6}$ show the radial stress distribution $\sigma_{r}$ along the radial direction. Figures $\mathbf{7}$ and $\mathbf{8}$ show the circumferential stress $\sigma_{\theta}$ along the circumferential direction.

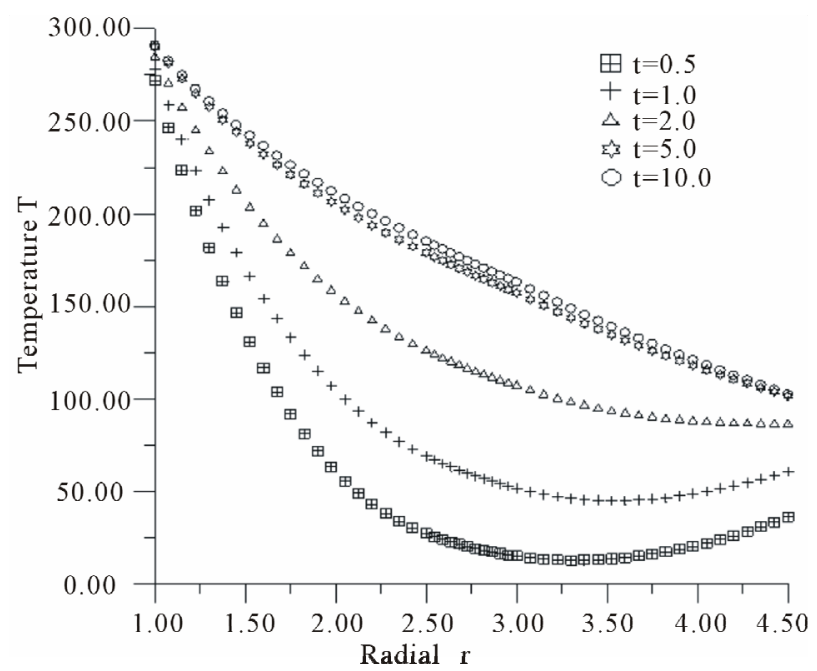

Figure 1. Temperature distribution along radial direction for 3 layers case.

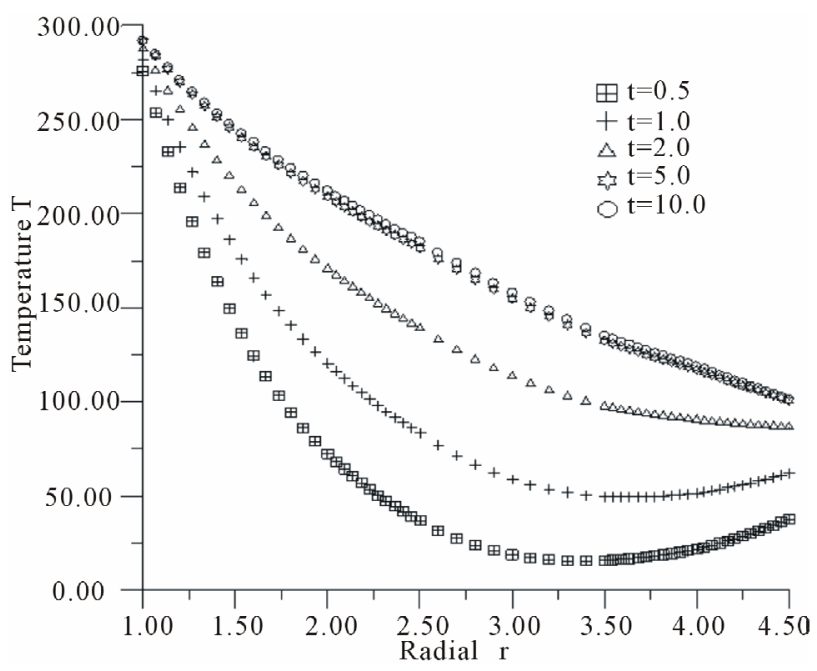

Figure 2. Temperature distribution along radial direction for 5 layers case.

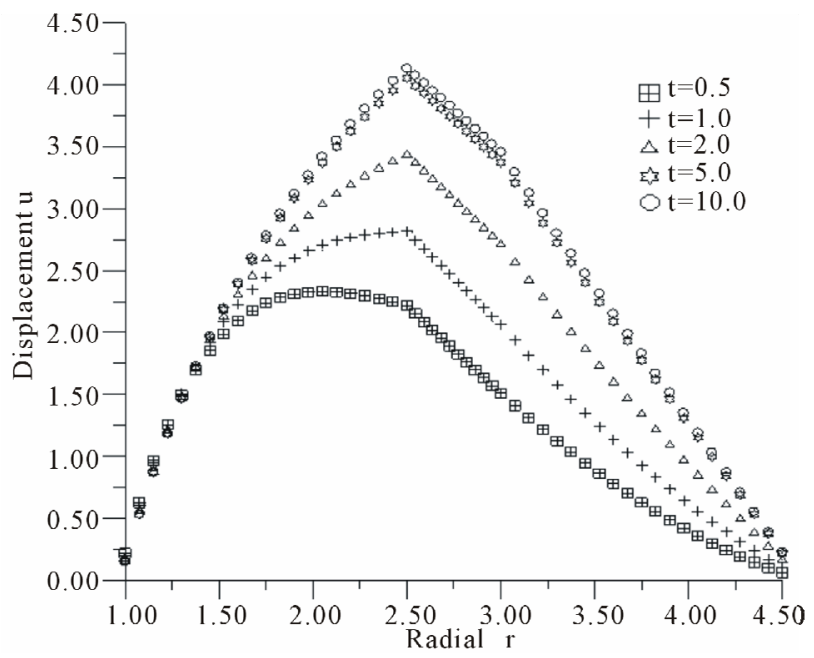

Figure 3. Radial displacement distribution along radial direction for 3 layers case.

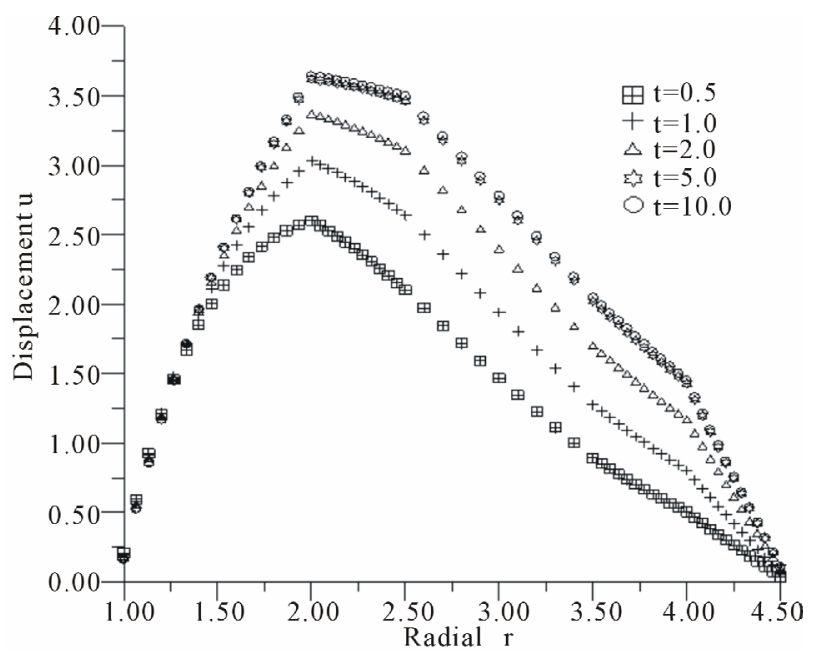

Figure 4. Radial displacement distribution along radial direction for 5 layers case.

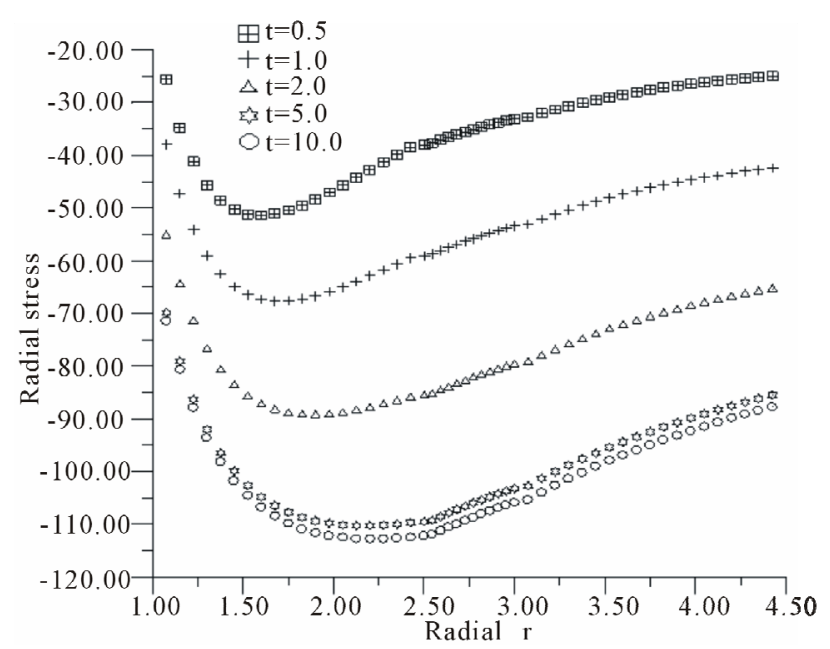

Figure 5. Radial stress distribution along radial direction for 3 layers case. 


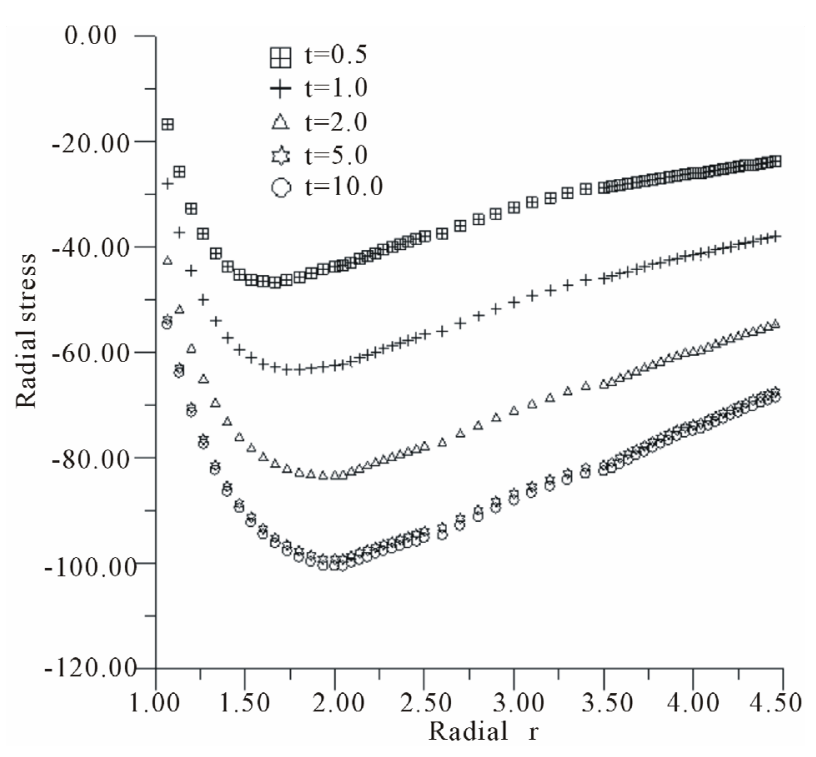

Figure 6. Radial stress distribution along radial direction for 5 layers case.

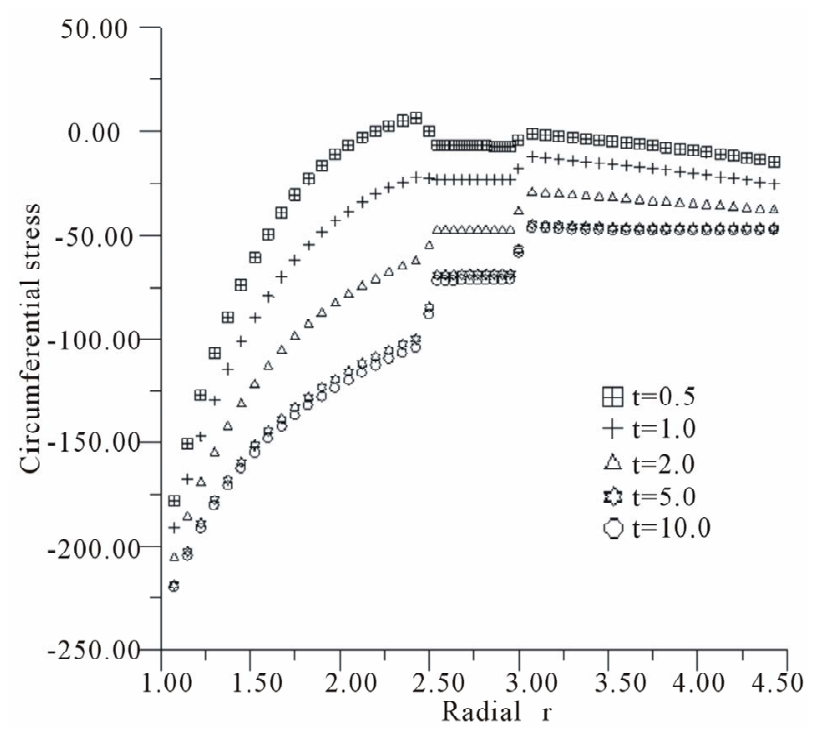

Figure 7. Circumferential stress distribution along radial direction for 3 layers case.

A method based on the finite difference and Laplace transformation has been developed to obtain numerical results. The temperature, displacement and thermal stress

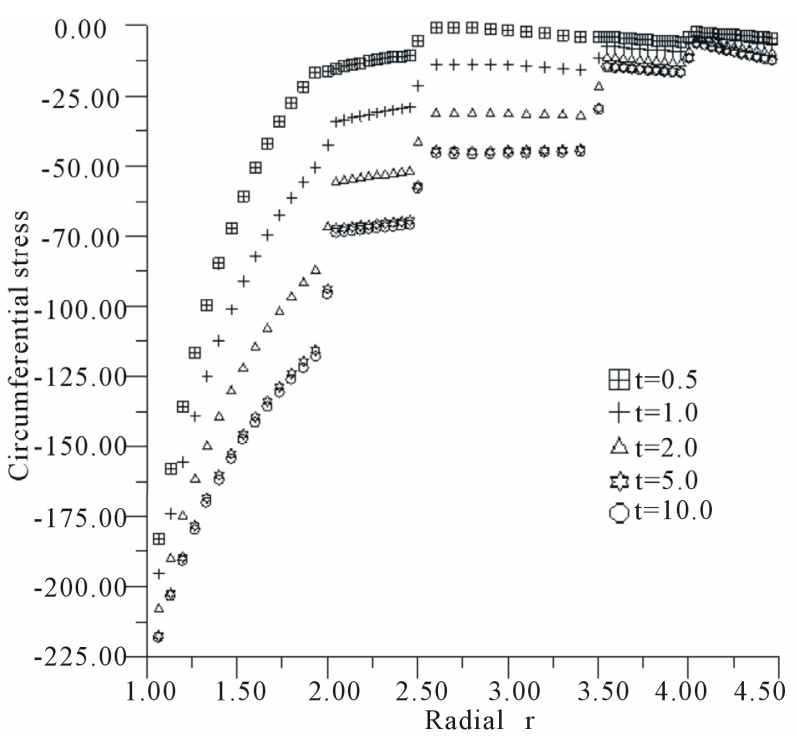

Figure 8. Circumferential stress distribution along radial direction for 5 layers case.

distributions have been obtained, all of which can be used to design useful structures or machines for engineering applications. There is no limit to the number of annular layers in a cylinder. Exemplifying numerical results from three- and five- layered cylinders at different time steps have been presented. The discontinuity in circumferential stress at each interface was found. It was found that the temperature distribution, the displacement and the thermal stresses vary slightly as the time increases.

\section{REFERENCES}

[1] H. H. Sherief and M. N. Anwar, "Problem in Generalized Thermoelasticity," Journal of Thermal Stresses, Vol. 9, No. 2, 1986, pp. 165-181. doi:10.1080/01495738608961895

[2] H. H. Sherief and M. N. Anwar, "A Problem in Generalized Thermoelasticity for an Infinitely Long Annular Cylinder Composed of Two Different Materials," Acta Mechanica, Vol. 80, No. 1-2, 1989, pp. 137-149. doi:10.1007/BF01178185 